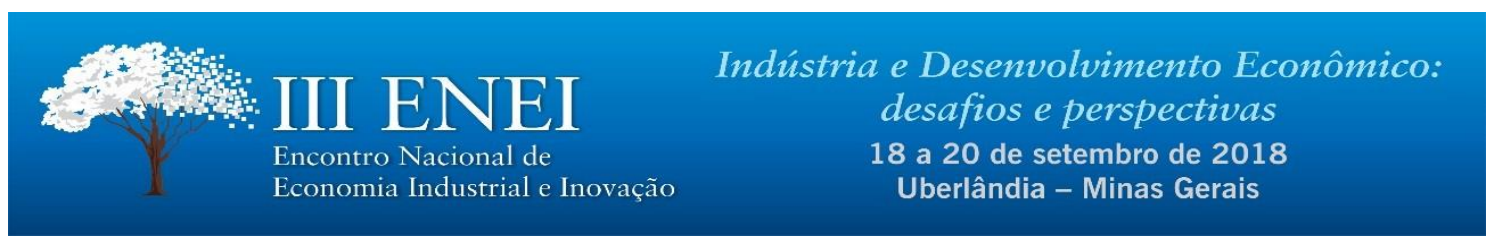

\title{
Capacidade de Absorção de Firmas e Interação Universidade-Empresa no Setor de Software do Estado de Santa Catarina
}

\author{
Luciana S. C. Vieira da Silva*1 \\ Silvio A. F. Cario**2 \\ Janaína Ruffoni***3
}

\begin{abstract}
Resumo
O presente estudo tem como objetivo analisar a interação universidade-empresa para o desenvolvimento inovativo no setor de software em Santa Catarina. Utiliza-se a abordagem a respeito da capacidade de absorção de conhecimento externo como tratamento teórico. Foram selecionadas cinco grandes empresas atuantes no setor de software para como estudos de caso. Essas empresas estão localizadas emaglomerações produtivas de Florianópolis, Joinville e Blumenau. A metodologia empregada apresenta as seguintes características: abordagem qualitativa, seleção da amostra intencional e por acessibilidade e utilização de dados de fontes primária e secundária. Os resultados apontam que empresas adotam ações estratégicas voltadas à obtenção de conhecimento externo junto às universidades. As pesquisas desenvolvidas em parceria com universidades resultam em aperfeiçoamento dos produtos existentes e na introdução de produtos novos pelas empresas no mercado. Universidades presentes nas localidades mencionadas, exercem o papel de elevar o nível de qualificação dos trabalhadores, cujos conhecimentos adquiridos auxiliam na construção da competência empresarial.
\end{abstract}

Palavras-Chave: Capacidade de Absorção; Interação Universidade-Empresa; Setor de Software de Santa Catarina/Brasil.

\begin{abstract}
The study aims to analyze the university-industry interaction for the innovative development in the software sector in Santa Catarina. The approach regarding the absorptive capacity of external knowledge is used as theoretical treatment. Five major firms in the software industry were selected as case studies. These firms are located in productive agglomerations of Florianópolis, Joinville, and Blumenau cities. The methodology used has the following characteristics: a qualitative approach, selection of the intentional sample and accessibility and use of data from primary and secondary sources. The results ind icate that companies adopt strategic actions aimed at obtaining external knowledge from universities. Research developed in partnership with universities result in the improvement of existing products and the introduction of new products by firms in the market. Universities present in the regions mentioned above play the role of raising the level of workers' qualification, whose acquired knowledge helps in the construction of entrepreneurial competence.
\end{abstract}

Key-Words: Absorptive Capacity; University-Industry Interaction, Santa Catarina's Software

\footnotetext{
1 * Profes sora do Departamento de Administração do Centro Universitário Municipal de São José - SC (USJ). Email: luvcosta@gmail.com

2 **Professor do Departamento de Economia e de Relações Internacionais e dos Programas de Pós-Graduação em Economia e Administração da Universidade Federal de Santa Catarina (SC). E-mail: fecario@yahoo.com.br

$3 * * *$ Professora do Programa de Pós-Graduação em Economia da Universidade do Vale dos Sinos - Porto Alegre (RS). Email:jruffonni@unisinos.br
} 
Sector/Brazil.

Área ABEIN: 4.4 - Redes de inovação

JEL Code: L20; L86; e O30. 


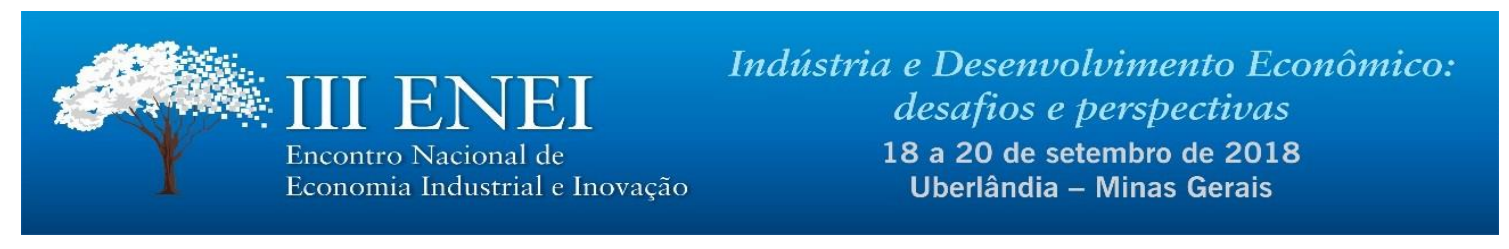

\section{Capacidade de Absorção de Firmas e Interação Universidade-Empresa no Setor de Software do Estado de Santa Catarina}

\section{Introdução}

O conhecimento constitui um dos principais insumos para o desenvolvimento inovativo. Através deste, criam-se condições para modificar produtos, processos, gestão e outros requerimentos voltados em melhorar o funcionamento do sistema econômico. A sociedade, por seu turno, beneficia-se dos avanços provocados pelo conhecimento de diferentes formas, dentre as quais o acesso aos produtos com melhor qualidade e menor custo, rapidez no processo de fabricação e na disposição dos produtos no mercado, na diferenciação e diversificação produtivas em atendimento a distintos mercados.

O conhecimento, por sua vez, requer cond ições para obtê-lo e trans forma-lo no propósito de atender determinados objetivos. Condições estas, que não são somente internas às empresas, mas depende da capacidade que se tem de adquiri-lo externamente. Nesta perspectiva, vem-se desenvolvendo nas últimas décadas tratamento teórico-analítico que procura entender como as empresas absorvem conhecimento externo, no propósito de gerar melhores condições organizacionais, produtivas, inovativas, enfim, competitivas no mercado.

Um importante espaço externo que as empresas buscam adquirir, assimilar, transformar e explorar o conhecimento é a universidade. No exercício de suas funções, a universidade cria, transfere e difunde o conhecimento para a sociedade. Contudo, a universidade não se encontra isolada de outros integrantes do sistema econômico. Depende de cond ições estruturais e organizacionais para o exercício de suas funções. Logo, quanto melhores forem as condições de funcionamento e os arranjos institucionais, maiores são as condições de a universidade contribuir para o meio empresarial ter acesso à mão-de-obra qualificada, solução de problemas técnicos, desenvolvimento de novos produtos, melhorias nos processos produtivos, etc.

No desenvolvimento das atividades econômicas, há segmentos que dependem mais de conhecimento externo. Há os segmentos econômicos que são baseados em ciência, cujas atividades encontram-se constantemente alimentadas por novos saberes, aprendizagens e ideias. Neste grupo se encontram, por exemplo, os segmentos eletrônico, química e software. Para estes, o conhecimento é complexo, de difícil acesso, de uso não difundido e em constante modificação. Logo, empresas realizam esforços para estarem em linha com o estado das artes do conhecimento, seja capacitando suas fontes internas (pessoal, laboratório e equipamentos) seja aproximando de fontes externas geradoras (fornecedores, clientes, consultorias e universidades).

Em Santa Catarina, o setor de software encontra-se em franco desenvolvimento. De acordo com a Federação das Indústrias de Santa Catarina - FIESC (2015), as empresas de alta tecnologia têm crescido, nos últimos anos, a taxas anuais de até $20 \%$. Tais empresas, cerca de 1.980, encontram-se localizadas em Blumenau, Chapecó, Criciúma, Florianópolis e Joinville, possuem faturamento superior a R 3 bilhões e geram mais de 20.000 empregos diretos. Atualmente, 78 empresas obtêm Portaria do Ministério da Ciência Tecnologia e Inovação (MCTI) para utilizar os benefícios fiscais da Lei de Informática. Destas, 24 empresas se beneficiam de isenção fiscal pela atividade desenvolvida e possuem o reconhecimento pelos produtos criados, como Bens Desenvolvidos no País.

O setor de software figura entre os principais segmentos de alta tecnologia e encontra-se fortemente concentrado em três localidades: Blumenau, Joinville e Grande Florianópolis. Esta última possui a maior concentração em número de empresas (187) quando comparada a Blumenau (106) e Joinville (80). Em relação às pessoas empregadas, Blumenau e Joinville contam com maior número atuando em softwaresob 
encomenda (1.462 e 1.783, respectivamente) e não-customizável (900 e 424, respectivamente) do que Florianópolis (1.207, sob encomenda; e 793, não-customízável).

Ao redor destas empresas existe uma estrutura de ensino, pesquisa e extensão universitária que contribui para o desenvolvimento de suas atividades. Em destaque, figuram na região da Grande Florianópolis, dentre outras a Universidade Federal de Santa Catarina (UFSC), Universidade para o Desenvolvimento do Estado de Santa Catarina (UDESC), a Universidade do Sul Catarinense (UNISUL) e a Universidade do Vale do Itajaí (UNIVALI), E, nas localidades de Joinville e Blumenau, destacam-se a Universidade da Região de Joinville (UNIVILLE) e a Universidade Regional de Blumenau (FURB), respectivamente, além de contarem com campus da UFSC e da UDESC. Outras unidades de completam a estrutura de ensino superior, entre estas a Pontifícia Universidade Católica do Paraná (PUCPR), em Joinville, e o Instituto Federal de Santa Catarina (IFSC), em Blumenau.

Considerando que o setor de software é classificado entre os que baseiam seu desenvolvimento a partir da ciência, pretende-se verificar neste trabalho, como as empresas do setor de software de Santa Catarina recorrem às universidades, como fonte de conhecimento externo, para atendimento de suas demandas. Para tanto, o trabalho encontra-se dividido em seis seções, sendo que nesta $1^{a}$ seção, expõe-se o objetivo principal; na $2^{\mathrm{a}}$ seção apresenta-se o tratamento teórico-analítico; na $3^{\mathrm{a}}$ seção descreve-se a metodologia; na $4^{\mathrm{a}}$ seção apontam-se as principais características da empresas selecionadas; na $5^{\mathrm{a}}$ seção analisam-se as formas de interação universidades-empresa no setor de software estadual; e, por fim, na $6^{\mathrm{a}}$ seção, faz-se a conclusão.

\section{Capacidade de Absorção e a Interação Universidade-Empresa}

\subsection{Capacidade de Absorção: significado e classificação}

Entende-se por capacidade absorção a exploração da aquisição, a assimilação e transformação de conhecimentos para gerar vantagem competitiva (EISENHARDT e MARTIN, 2000; ZAHRA e GEORGE, 2002). Nesta perspectiva, a capacidade de absorção refere-se aos procedimentos para analisar a acumulação e o fluxo de conhecimento em uma empresa (CHANG, HOU e LIN, 2013).

O conceito de capacidade de absorção está embutido no debate sobre as capacidades dinâmicas que foram originadas da teoria evolucionária da empresa. (NELSON; WINTER, 1982). Estas últimas permitem as empresas reagirem às mudanças no ambiente de negócios e evitarem a obsolescência das competências (TEECE; PISANO, 2007).

Teoricamente, o conceito de capacidade absorção está localizado entre os campos de aprendizagem organizacional (HUBER, 1991; KIM, 1998), a gestão do conhecimento (CHIVA; ALEGRE, 2005; OSHRI; PAN; NEWELL, 2006) e as capacidades dinâmicas (MOWERY; OXLEY; SILVERMAN, 1996). Existem estudos que pensam na capacidade de absorção como a capacidade dos indivíduos e de empresas (COHEN e LEVINTHAL, 1990) ou países (MOWERY; OXLEY, 1995).

A ideia da habilidade da empresa adquirir conhecimento do ambiente externo não foi originado por Cohen; Levinthal (1989). Como os autores mencionam na introdução do seu artigo de seminal, vários outros autores fizeram observações similares. Mowery (1983) realizou apontamentos similares sobre Pesquisa e Departamento (P\&D) interno e assimilação do conhecimento externo, em suas discussões de gestão de P\&D, em múltiplas indústrias.

De acordo com Lane, Koka e Pathak (2006), o que Cohen e Levinthal adicionam à literatura emergente foi um rico conjunto de explicações baseado na economia da organização industrial, de como e porque o $\mathrm{P} \& \mathrm{D}$ da empresa tem um papel secundário, explicações apoiadas em testes empíricos e um nome conciso e evocativo para aquele papel, ao qual chamaram de capacidade de absorção.

Através das atividades de $\mathrm{P} \& \mathrm{D}$, a empresa desenvolve conhecimento organizacional sobre certas áreas da ciência e tecnologia, e como essas se relacionam aos produtos e mercados (capacidade de identificar e valorizar o conhecimento, Cohen; Levinthal, 1989). Com o tempo, a empresa desenvolve processos, políticas e procedimentos que facilitam o compartilhamento do conhecimento interno (capacidade de assimilar o conhecimento externo, Cohen; Levinthal, 1990). Torna-se capaz de fazer uso deste último para prever tendências tecnológicas, criar produtos e mercados e pensar estrategicamente 
(capacidade de assimilar o conhecimento externo e aplicá-los comercialmente, Cohen; Levinthal, 1990 e 1994).

Nesta perspectiva, desenvolver e manter a capacidade de absorção é vital para a sobrevivência e sucesso a longo prazo de uma empresa, pois pode reforçar, complementar ou reorientar a base de conhecimento existente. Zahra e George (2002) consideram capacidade de absorção como "(...) um conjunto de rotinas organizacionais e processos pelos quais as empresas adquirem, assimilam, transformam e utilizam os conhecimentos para produzirem uma capacidade organizacional dinâmica". Argumentam que as empresas podem adquirir e assimilar conhecimentos, mas podem não ter a capacidade de transformar e explorar os conhecimentos na obtenção de lucros.

Em contribuição a este tratamento teórico-analítico, Zahra e George (2002) classificam capacidade de absorção potencial e capacidade de absorção realizada. A primeira capta a aquisição e assimilação de conhecimento, condição que permite à empresa identificar e adquirir conhecimento gerado externamente. Enquanto que a segunda, refere-se à capacidade de transformar e explorar os conhecimentos adquiridos para fins comerciais.

A literatura é contraditória na sequência do tratamento sobre a absorção do conhecimento. Zahra; George (2002) e Jansen; Van den Bosch; Volberda (2005) conceitualizam a sequência da ab sorção, como uma relação linear entre a aquisição, assimilação, transformação e exploração. Enquanto, Todorova; Durisin (2007) interpretam a assimilação e a transformação como dois elementos paralelos. O conhecimento é assimilado, se a estrutura cognitiva existente dos membros da organização não mudar. E, a transformação ocorre se os novos conhecimentos estiverem inter-relacionados com a mudança de estruturas cognitivas existentes.

Examinando diferentes efeitos de antecedentes organizacionais, as capacidades de absorção potencial e realizada não só esclareceram como a capacidade de absorção pode ser desenvolvida, mas também revelaram por que as empresas têm dificuldades em gerir as dimensões da capacidade de absorção com sucesso. (JANSEN, VAN DEN BOSCH, VOLBERDA, 2005).

A capacidade de absorção potencial reflete a aquisição e assimilação, enquanto que a capacidade de absorção realizada incorpora a noção de Kim (1998) sobre a capacidade de transformação da empresa. Nesta perspectiva, a capacidade de desenvolver e de refinar rotinas facilita a combinação do conhecimento existente, com o conhecimento recém-adquirido e assimilado. Esta forma de capacidade de absorção determina a taxa e efetividade ao qual o conhecimento adquirido, via alianças exploratórias, pode ser internalizado (XIA; ROPER, 2008).

Independentemente da sequência de assimilação e transformação de conhec imento, as capacidades de absorção potencial e realizada estão ligadas através de um fator de eficiência. Neste contexto, um fator de eficiência mais elevado conduz a um maior desempenho da inovação, porque as organizações perseguem um curso de ação em resposta ao seu conhecimento potencial (ZAHRA; GEORGE, 2002). De acordo com o fator de eficiência, Winter (2000) sugere que a satisfação, ao invés da otimização, orienta os gestores na transformação de conhecimento potencial em realizado.

Van den Bosch; Volberda, Boer (1999) assumem que o conteúdo e o processo de absorção de conhecimento de uma empresa podem ser analisados utilizando três dimensões da absorção do conhecimento. Estas dimensões são eficiência, alcance e flexibilidade. Eficiência de absorção de conhecimento refere-se à forma como as empresas identificam, assimilam e exploraram o conhecimento a partir da perspectiva de custo e economias de escala. Alcance de absorção de conhecimento refere-se à amplitude de conhecimento em que se baseia uma empresa. E, flexibilidade de absorção de conhecimento refere-se até que ponto uma empresa pode acessar conhecimento adicional, e reconfigurar o existente.

Mowery, Oxley, Silverman (1996) afirmam que muitos pesquisadores veem a capacidade de absorção sob duas perspectivas gerais: como estoque de conhecimentos relacionados previamente e na competência de absorver o conhecimento externo. A primeira apresenta-se como estoque, dado que leva em consideração o nível de conhecimento prévio relevante, presente na unidade focal. Em verdade, tratase da base de conhecimento da empresa, correspondente ao nível que possui no tempo. Enquanto a segunda, apresenta-se como expressão da competência que a empresa tem de absorver o conhecimento que está além de seus limites estabelecidos. 
Segundo Liao, Wu, Hu e Tsuei (2009), estudos relacionados à capacidade de absorção apresentam elementos centrais relacionados ao: $1^{\mathrm{a}}$.) conhecimento interno incluindo capital humano e tecnologia; $2^{\mathrm{a}}$ ) ambiente externo, tais como políticas e regras governamentais, interações industriais e risco; $3^{\mathrm{a}}$.) gastos em $\mathrm{P} \& \mathrm{D} ; 4^{\mathrm{a}}$.) intensidade de aprendizagem interna; $5^{\mathrm{a}}$.) estraté gias organizacionais; e, $6^{\mathrm{a}}$.) processos ino vativos como busca de vantagem competitiva. Tais elementos sintetizam o corpo dos estudos relacionados à capacidade de absorção de conhecimentos externos para serem aproveitados pelas empresas, internamente, no cotidiano de suas atividades.

\subsection{Interação Universidade-Empresa: aspectos interativos dos meios acadêmico e empresarial}

A universidade figura como espaço em que se cria, desenvolve e transfere conhecimento para a sociedade. Para Nelson (2006, p. 124) esta é “(...) reconhecida como o repositório dos conhecimentos científicos e tecnológicos públicos, conhecimentos estes que elas criam e reproduzem através do ensino e vão aumentando por meio da pesquisa". O exercício destas funções não ocorre de forma isolada; desenvolver-se em interação com o ambiente que a cerca. Figura como integrante de um sistema de inovação de um país, composto de empresas, fornecedores, consumidores, institutos de pesquisa, bancos e governo entre outras instituições, para desenvolver e trocar conhecimento e informação em favor de inovações (EDQUIST, 1997 e 2001).

Como observa Lee (2000) a universidade colabora com as empresas de diferentes formas com destaque para resolver problemas técnicos, desenvolver novos produtos e processos, melhorar a qualidade dos produtos existentes, reorientar a agenda de $\mathrm{P} \& \mathrm{D}$, realizar pesquisa em direção à novas patentes, ter acesso às novas pesquisas através de seminários e workshops; recrutamento de recursos humanos qualificados, entre outros. Segatto; Sbragia (2002) apontam que arranjos interativos melhoram a competitividade das empresas, na medida em que as pesquisa realizadas se traduzem em vantagens competitivas, postas em aumento dos rendimentos e menores custos e riscos empresariais.

Lemos (2013) sintetiza os resultados dos trabalhos de Meyer-Krahmer; Schmoch (1998), Cohen et al (2002) e Perkmann; Walsh, (2007), demonstrando as várias dimensões da interação universidadeempresa. O primeiro aponta que interações ocorrem a partir de pesquis a colaborativa, contatos informais, formação de pessoal, elaboração de teses, contrato de pesquisa, conferências, consultoria, seminários, intercâmbio de cientistas, publicações e comitês. O segundo registra que as interações ocorrem através de publicações, reuniões, conferências, contrato de pesquisa, consultoria, empreendimentos, patentes, licenças e contratação de graduados. E, o terceiro, destaca links através de parceria de pesquisa, execução de serviços, empreendedorismo, transferência de recursos humanos, interação informal, comercialização de direitos de propriedade e publicações.

Inúmeros trabalhos procuram apresentar os benefícios que as empresas obtêm a partir da interação como meio acadêmico universitário. Betti; Santoro (2011) destacam a capacidade de aprender no propósito de melhorar competência para buscar novas tecnologias. Dutrénit; Arza (2010) apontam a implementação de estratégias de inovação de longo prazo, a partir das capacitações construídas. Mueller (2006), Bischop, D'Este e Neely (2011) sinalizam a exploração de novos conhecimentos que alimentam ideias que promovem mudanças técnicas e organizacionais. E, para Meyer-Kramer; Schmoch (1998) os vínculos elevam a capacidade empresarial de resolução dos problemas técnicos e para absorver pessoas mais qualificadas para seus quadros.

Por seu turno, as universidades também obtêm benefícios decorrentes da interação com o meio empresarial. Para Lee (2000), as universidades podem testar e aplicar na prática a própria teoria que desenvolvem, obter conhecimentos mais aprofundados na área de pesquisa, ter acesso a financiamento que complementa os recursos públicos e, criar canal de acesso para colocação profissional de estudantes. $\mathrm{O}$ estudo de Arza (2010) cita elementos do trabalho anterior e incorpora a reputação acadêmica para o pesquisador e à instituição de ensino. E, Alvin (1998) agrega aos itens citados, o exercício de função social e a atualização dos currículos acadêmicos.

As interações envolvendo empresas e universidade apresentam intensidades diferentes. Malerba e Orsenigo (1993) afirmam que a tecnologia conta com as propriedades de oportunidade tecnológica, cumulatividade do conhecimento, apropriabilidade dos resultados e de conhecimento base. Cassiolato et al 
(1996) analisam os vínculos das empresas com universidades, a partir destas propriedades e do padrão tecno lógico setorial de Pavitt (1990). Empresas dos setores baseados em ciência - química, eletrônica, software, por ex. - em virtude da dinâmica tecnológica, devem se aproximar de forma intensa do meio acadêmico para atualização e ampliação do conhecimento. Diferentemente, de empresas dos setores dominados por fornecedores, intensivos em escalas e fornecedores especializados que possuem menos interações, em face da tecnologia ser de uso difundido, não complexa e de acesso não-restrito.

Salienta-se, ainda, que as interações universidade-empresa são marcadas por barreiras que impedem melhor relacionamento. Dentre as principais, Brunnel, D'este; Salte (2010), Arza (2010) e Segatto; Sbragia (2002) apontam: diferença de orientação envolvendo o objetivo da universidade pela pesquisa básica e a empresa pela pesquisa aplicada; temporalidade distinta da pesquisa, dado que a empresa volta-se para resultados no curto prazo, e a universidade de longo prazo; incentivos diferentes que movem os profissionais das empresas e da universidade, o primeiro guiado pela lógica do valor de mercado e o segundo pelos valores científicos; os pesquisadores universitários interessados na divulgação dos resultados nos meios de comunic ação e o profissionais das empres as querendo confidencialidade; e diferenças de nível de conhecimento das pessoas das universidades e da empresa envolvida na interação.

\section{Aspectos Metodológicos}

A pesquisa contempla o estudo de cinco empresas do setor de software que estão localizadas no estado de Santa Catarina, mais especificamente em Florianópolis, Joinville e Blumenau. As empresas estudadas são consideradas de grande porte, tomando como referência o faturamento obtido e, na sua maioria, são líderes nos mercados em que atuam. Tais empresas desenvolvem sistemas ERP's (Enterprise Resource Planning), subsetor que obtém elevado faturamento e atende diversos segmentos de mercado.

A seleção da amostra ocorreu de forma intencional e por acessibilidade. Para atender o critério da intencionalidade utilizou-se a classificação de empresas de software feita por Fleury (2007). Nesse estudo, a classificação obedece ao critério: orientado ao cliente, orientado ao produto e orientado a serviço. Esta divisão representa melhor a realidade das empresas do setor, do que a feita pela Fundação Instituto Brasileiro de Geografia e Estatística (IBGE), dividida em software por encomenda, software customizável e software não customizável. A classificação do IBGE está mais voltada para questões contábeis do que pela atividade realizada e, por isso, seria difícil separar uma empresa que trabalhe com software customizável sob encomenda, por exemplo. Na taxonomia eleita por Fleury (2010), a empresa pode ser classificada numa das categorias mencionadas, uma vezque a variedade de produtos/serviços é levada em consideração.

O critério de acessibilidade depende do contato para se ter acesso ao gestor da empresa e da disponibilidade destes concederem a entrevista. Em algumas empresas, o acesso ocorre por meio de indicação do gestor e a liberação do contato pelo mesmo. Vale ressaltar que algumas empresas não permitem a divulgação do nome, e para fins de não identificação, estas foram renomeadas, conforme o Quadro 1.

Quadro 1: Empresas entrevistadas do setor de software de Santa Catarina, 2016

\begin{tabular}{|l|l|l|l|l|}
\hline Empresa & Localização & Porte Empresarial & Cargo do Entrevistado & $\begin{array}{l}\text { Data da } \\
\text { Entrevista }\end{array}$ \\
\hline Alfa & Florianópolis & Grande Empresa & Diretor de Marketing & $25 / 08 / 2016$ \\
\hline Gama & Florianópolis & Grande Empresa & Asses sor de Projetos Especiais & $16 / 09 / 2016$ \\
\hline Ômega & Blumenau & Grande Empresa & Coordenador de Inovação & $05 / 10 / 2016$ \\
\hline Delta & Joinville & Grande Empresa & Diretor de Projetos & $31 / 10 / 2016$ \\
\hline Lambda & Blumenau & Grande Empresa & Diretor de Desenvolvimento de Software & $07 / 11 / 2016$ \\
\hline
\end{tabular}

Fonte: Elaborado pelos autores

A opção por amostras de caráter não probabilístico ou não aleatório justifica-se, principalmente, pela característica qualitativa da pesquisa e pela dificuldade de acessibilidade. $\mathrm{O}$ tratamento dos dados ocorre por meio de análise documental e de conteúdo. A presente pesquisa considera dois conjuntos de 
conteúdos de informação, a saber: i) dados primários obtidos por meio das entrevistas; e, ii) informações disponíveis nos sites das empresas entrevistadas.

\section{Principais Características das Firmas Selecionadas do Setor de Software do Estado de Santa Catarina}

A empresa Alfa, criada em 1990, encontra-se localizada em Florianópolis. Iniciou com três sócios, à época funcionários do Centro de Informática e Automação de Santa Catarina (CIASC). Estes, após a jornada de trabalho nesta empresa desenvolviam software de gestão, como os relacionados ao controle de caixa, gerenciamento de clínicas, entre outros. No momento, trata-se de uma das maiores empresas do Brasil voltada ao desenvolvimento deste segmento de softwares, com atuação em vários estados, vários países da América Latina e nos Estados Unidos.

Desde 1990, a empresa Alfa atua na área de gestão pública e privada, se posicionando com tecnologias modernas e inovadoras. Possui cerca de 2.800 clientes, conta com mais de 1.500 colaboradores e tem um faturamento médio anual de $\mathrm{R} \$ 70$ milhões. Ao longo desses anos, esta empresa se especializou no desenvolvimento e na implantação de softwares de gestão para os segmentos de justiça, infraestrutura, gestão pública e construção civil. Dentre as inovações geradas, destaca-se uma patente registrada de software para construção civil.

A empresa Gama, também localizada em Florianópolis, inicia suas atividades fornecendo produtos para telecomunicações, em 1981 cria o serviço de despertador, e em seguida, o de informações por telefone utilizado em todo o território brasileiro. Com o período de privatizações do Governo Fernando Henrique Cardoso, a empresa passa a investir em informática e, em 1998 desenvolve o serviço de escuta telefônica em substituição ao "grampo telefônico". Seu portfólio de produtos atual inclui software e hardware de Inteligência Corporativa e Investigativa, Call Center e Contact Center, Convergência de Redes e PABX, além de serviços em tecnologia de informação e telecomunicações. Comercializa plataformas de Computer \& Telephone Integrated (CTI) e para redes corporativas e órgãos de segurança pública.

O faturamento anual da empresa é de cerca de R\$ 100 milhões e conta com 684 funcionários, sendo que $75 \%$ estão alocados na atividade fim da empresa. Possui escritórios em Brasília, Curitiba, Florianópolis, Fortaleza, Porto Alegre, Recife, Ribeirão Preto, Rio de Janeiro e São Paulo, além de atuar na Argentina, Colômbia, Costa Rica, Equador, México, Moçambique, Panamá, Paraguai, Peru, Uruguai e Venezuela. Realiza parcerias com instituições de ensino superior, sendo destaque as interações com a UFSC e a Universidade Federal do Ceará (UFCE). Possui dois registros de patentes: analisador digital de sinais multifrequências e equipamento para mala direta.

A empresa Delta, situada em Blumenau, encontra-se atuando no setor de software há 29 anos. O curso de graduação em ciências da computação da FURB e a demanda crescente de empresas locais pelos seus produtos auxiliam no desenvolvimento desta empresa. A Delta trabalha com softwares de gestão empresarial nos segmentos de softwares de gestão de pessoas, acesso a segurança e de logística, bem como desenvolve softwares de performance corporativa, os "BI's", workflows e Dashboard.

Possui unidades de negócio situadas nas cidades de Indaiatuba (SP), Porto Alegre (RS) e Joinville (SC) e cerca de 100 canais de distribuição pelo país. A empresa realiza, no curso de suas atividades, parcerias com institutos de pesquisa como a Fundação Centro de Referência em Tecnologias Inovadoras (CERTI) e Universidades, dentre as quais a UNISUL, a UFSC e a FURB, no propósito de desenvolver P\&D no modelo de inovação aberta. A unidade de Blumenau possui entre 1.200 e 1.300 funcionários, mas ao todo são 3.000 pessoas envolvidas entre colaboradores, parceiros comerciais e canais de distribuição. A empresa conta com cerca de 10 mil clientes e o faturamento anual, do grupo que pertence, situa-se em torno de $\mathrm{R} \$ 56$ milhões.

A empresa Ômega é fruto da fusão de ema empresa localizada em São Paulo (SP) e a outra em Joinville (SC). A empresa de São Paulo iniciou suas atividades em 1983, desenvolvendo softwares e computadores pessoais para pequenas e médias empresas. A empresa de Joinville nasceu em 1992, desenvolvendo softwares empresariais. Em 2005, ocorre a fusão entre as empresas, contando com o apoio do BNDES. Em 2008, foi feita a aquisição de outra empresa de grande porte de Joinville (SC). Em 2011, criou a Ômega Labs no centro de pesquisa de soluções em computação em nuvem, "cloud computing", no 
Campus da Universidade da Califórnia. Entre os anos de 2013 e 2014 adquiriu oito empresas de software no Brasil e uma outra nos EUA. A área de atuação volta-se para o desenvolvimento e comercialização do direito de uso de aplicativos para gestão empresarial, Enterprise Resource Planning (ERP). Além disso, comercializa serviços de adaptação dos softwares a pedido de cliente e realiza implantação, treinamento, consultoria e manutenção de sistemas.

Esta empresa possui elevada participação no mercado de software de gestão na América Latina, $34,5 \%$, e detém a liderança no Brasil, com $48,6 \%$ de fatia do mercado. O faturamento anual gira ao redor de $\mathrm{R} \$ 1,7$ bilhão. A empresa dispõe de seis filiais, além de 52 franquias e mais de 40 canais alternativos de distribuição no Brasil. No mercado internacional, atua em 15 países da América Latina, possui uma filial em Portugal e tem uma franquia na África. A empresa Ômega possui 9.000 funcionários, sendo $1.100 \mathrm{em}$ Joinville, dos quais 200 dedicados à área de inovação. Conta com patente registrada no Instituto Nacional de Propriedade Industrial (INPI) desde 2007, referente à métodos e sistemas para ciclo de vendas e de implementação de software.

A empresa Lambda, iniciou suas atividades e 1997 e encontra-se localizada em Blumenau. No início, sua atuação voltou-se ao desenvolvimento de software de gestão empresarial $(E R P)$, e atualmente oferece soluções em software, serviços e gestão de processos adminis trativos. Neste curso, especializou-se em sistemas e serviços complementares para as áreas: jurídica, recursos humanos e gestão de viagens. A empresa também cria soluções nas áreas de logística e supply chain, turismo e de saúde - operadoras e hospitalar - com foco em tecnologia, gestão de sinistro, terceirização e serviço de orientação médica (nurse call). Comercializadas no modelo tradicional e em Cloud Computing e SaaS (Service as Service).

A empresa Lambda procura realizar atendimento próximo e o desenvolvimento de serviços personalizados, baseados em Service Level Agreement - (SLA). Contando com cerca de 1.200 colaboradores, figura entre os cinco maiores players nacionais no mercado de software de gestão empresarial no Brasil. Possui dois centros de desenvolvimento de tecnologia e escritórios localizados em Alphaville, São Paulo (SP), em Blumenau (SC), Curitiba (PR), Maringá (PR) e Rio de Janeiro (RJ), além de canais de venda nos principais polos econômicos nacionais. Em 2015, o grupo que pertence a empresa Lambda registrou faturamento da ordem de $\mathrm{R} \$ 195$ milhões.

\section{Interação Universidade-Empresa no Setor de Software em Santa Catarina}

\subsection{Especificidades das Interações}

A empresa Alfa considera relevante as interações entre os atores pertencentes ao sistema inovação para seu êxito. Os vínculos reinantes com empresas privadas, institutos de pesquisa e/ou universidades criam condições para o estabelecimento de projetos conjuntos, troca de informações, transferência de conhecimento, contratação de pessoal qualificado, entre outros aspectos que possibilitam desenvolver inovações. A afirmação de um entrevistado respalda esta visão: "Nós temos parcerias de fornecimento de tecnologia com os grandes players mundiais, Oracle, IBM, também com universidades, principalmente com a UFSC que é o principal parceiro".

As parcerias que a empresa Alfa realiza com universidades são mais intensas na formação de mãode-obra qualificada para atuar na empresa. A UFSC é, em grande monta, responsável pela formação qualificada de pessoas atuantes na área de software da região da Grande Florianópolis. No entanto, também são realizadas parcerias na área de pesquisa, sobretudo com esta universidade ao qual a empresa mantém relações, conforme relato do entre vistado: “(...) então hoje a maior parte das parcerias que nós temos com as Instituições de Ensino é para a formação de mão de obra qualificada pra atuar na empresa, mas também tem algumas parcerias tecnológicas com a UFSC."

Uma adaptabilidade proativa da empresa ocorre ainda na década de 1990 quando o conceito de “Cloud Computing" ainda não era uma realidade no Brasil. Com o propósito de desenvolver pesquisa nesta área, estabeleceu parceria com a UFSC, voltada em proporcionar maior segurança aos dados, possibilitando, assim, seus clientes postarem conteúdo na nuvem. Essa aposta deu à empresa o pioneirismo no mercado de ERP em nuvem, conforme declarou o entrevistado: “(...) nós temos 1400 empresas operando 
em nuvem, então em termos de Brasil não tem outra empresa assim e, em termos mundiais é um case bastante significativo".

Em 2007, o Instituto Stela, laboratório do Programa de Pós-Graduação em Engenharia de Produção (PGEP) da UFSC, requisita à empresa auxílio para a manutenção da plataforma que integra dados da Coordenação de Aperfeiçoamento de Pessoal de Nível Superior (CAPES), do Conselho Nacional de Desenvolvimento Científico e Tecnológico (CNPq) e da Universidade com conexão Web. Esta parceria constitui uma das primeiras experiências de inovação aberta do Instituto Stela, onde a empresa Alfa fornece ferramentas de gerenciamento de projetos, Business Intelligence (BI), gerenciamento eletrônico de documentos (GED) e geoprocessamento, permitindo ao Instituto aperfeiçoar o próprio sistema e ampliar suas oportunidades de negócio.

Em 2011, outra expressão desta parceria se manifesta pelo uso dos produtos da empresa. A UFSC torna-se cliente do software de gestão pública da empresa Alfa, cujo produto teve participação de pessoas formadas por esta instituição de ensino superior. A interação permite que ocorra participação mútua de pesquisadores da empresa e da universidade, na geração do produto.

Afirmações do representante da empresa Alfa apontam o reconhecimento da melhoria das cond ições competitivas, a partir dos relacionamentos firmados com a instituição de ensino superior. A troca de informações e conhecimentos estabelecido por meio da interação com laboratórios e seus pesquisadores cria condições para promoção de mudanças técnicas relevantes. Realça-se o papel da universidade como responsável pela formação de recursos humanos, dado que o conhecimento incorporado nas pessoas é utilizado para aperfeiçoamento e criação de produtos e serviços.

Na empresa Gama, a interação realizada com universidades, ocorre não só com o propósito de contratar profissionais formados e em formação dos cursos de ciências da computação, sistemas de informação, engenharia e outros da UFSC, mas também em parcerias realizadas com diversos laboratórios desta universidade para desenvolver pesquisa tecnológica.

Segundo o entrevistado, a universidade realiza pesquisa científica e a empresa oferece infraestrutura interna, para que o conhecimento gerado possa ser colocado em prática. O vínculo reinante possibilita a transformação das ideias em produtos para o mercado. Neste sentido, a empresa possui vínculos com a Fundação Centros de Referência em Tecnologias Inovadoras (CERTI), o Laboratório de Integração de Software e Hardware (LISHA) e o Laboratório de Circuitos e Processamento de Sinais (LINSE).

A interação com o laboratório LINSE da UFSC, constitui a maior referência de vínculo para a empresa entre o meio acadêmico e o meio empresarial. Esta parceria resultou, por exemplo, na criação de um taquígrafo que transcreve automaticamente o áudio, considerada importante inovação para o quadro dos produtos oferecidos pela empresa. A expressão abaixo enfatiza a importância do laboratório LINSE para a empresa:

\footnotetext{
"A e mpresa trabalha desde 90 como laboratório LINSE (Laboratório de Circuitos e Processamento de Sinais) da universidade (UFSC). Ela trabalha com outros CERTI, LISHA (Laboratório de Integração de Software e Hardware), etc, etc, mas o LINSE ela já tem uma relação muito longa, pra nós é o melhor laboratório que tem lá, que trabalha neste segmento".
}

Outras parcerias com universidades são realizadas nos últimos anos, como as firmadas com a Universidade Federal do Ceará (UFC) e o Serviço Nacional da Indústria (SENAI). Tais parcerias expressas no propósito de realização de pesquisa e de qualificação de trabalhadores, segundo entre vistado, visam criar melhores condições para o desenvolvimento inovativo interno.

Reforça o argumento empresarial para constante interação com o meio acadêmico, a existência de um setor interno de P\&D. Contar com infraestrutura tecnológica interna contribui para manter ativa a capacidade absortiva da empresa. Dada a existência desse laboratório específico interno ser de longo período, criou-se uma cultura organizacional voltada à busca de conhecimentos externos para capacitar as condições internas dedicadas ao desenvolvimento inovativo.

No setor de $\mathrm{P} \& \mathrm{D}$, os colaboradores são incentivados à realizar pesquisas em bases de dados de periódicos internacionais, a fim de identificar a tecnologia que está sendo cientificamente estudada no momento. Nesta rotina, os pesquisadores recorrem a bases de dados como Elsevier, Scopus, Science Direct 
e Thompson Reuters, no propósito de auxiliar na busca de soluções para os problemas tecnológicos da empresa. Neste sentido, o entrevistado relata:

“(...) vê-se qual é a tecnologia, faz-se o gap de análise, achou isso capacita a turma que vai fazer pelo que se achou. Então a turma de $P \& D$ tem que saber pesquisar. A turma que desenvolve a inovação tem que saber a tecnologia que essa turma que pesquisou descobriu”.

No processo de aquisição de conhecimento, a equipe, que realiza tal pesquisa, compõe a tecnologia base da empresa Gama voltada em compreender a linguagem de programação, constituição de banco de dados, participação nos projetos de placa, no firmware e de chip de computador. Além disso, outros meios de aquisição de conhecimento são utilizados como participação em eventos, feiras e palestras, consultorias.

A qualificação dos colaboradores ocorre através de incentivos para realizar cursos de graduação e pós-graduação em área de conhecimento vinculada ao core competence da empresa. Cerca de 60 a $70 \%$ do valor das mensalidades e outras despesas estudantis são pagas pela empresa Gama. Tal regime de incentivo possibilita que cerca de $70 \%$ dos colaboradores pos suam curso superior. Para a qualificação relacionada à realização de cursos no stricto sensu, como mestrado e doutorado, o incentivo da empresa se vincula a ceder o colaborador nos dias em que ele possui atividades acadêmicas, sem realizar descontos no salário.

A empresa Delta se gue orientação interna de se relacionar com universidades através da formação de mão-de-obra dos colaboradores. Para tanto, disponibiliza regime de incentivo por meio de concessão de bolsas de estudo e de isenção de horas de trabalho, enquanto estiver frequentando curso superior. $\mathrm{O}$ entrevistado relata como ocorre a interação da empresa com as Universidades:

\footnotetext{
"O acesso [...] ao conhecimento com [...] a universidade [...] vem muito como formação de mãode-obra de funcionário. Então assim, [...] tem um programa que apoia o funcionário com bolsas de até $50 \%$ para que eles possam estudar em universidades privadas. Em universidades públicas também tem o apoio, mas é com a isenção de horas de trabalho, se ele precisar [...]".
}

A exploração de linhas de incentivo também se estende através de obtenção de recursos do CNPq, no programa de Formação de Recursos Humanos em Áreas Estratégicas (RHAE). A afirmação do entrevistado desta empresa referenda esta observação: "No passado a gente teve uma experiência com o $C N P q$ utilizando o pesquisador RHAE”. Este programa consiste em possibilitar que pesquisadores da empresa obtenham títulos de mestrado e de doutorado junto a universidades de referência, no intuito de obter maior qualificação acadêmica para atuarem nas áreas de P\&D da empresa.

Além desta fonte de financiamento, a empresa recorre ao regime de incentivo da Lei do Bem e das linhas de financiamento do Banco Nacional de Desenvolvimento Econômico e Social (BNDES) para P\&D, como forma de desenvolver o potencial tecnológico interno. Neste contexto, buscam-se recursos com o intuito de melhorar as condições física, técnica e pessoal da infraestrutura laboratorial. A expressão a seguir suporta esta observação: “O maior benefício, digamos assim, no P\&D da empresa vem da Lei do Bem e das linhas de financiamento do BNDES". Com tal empreendimento, criam-se condições para maior aproximação dos meios empresarial e acadêmico em trocas de informação, geração de conhecimento e formas de aprendizado.

A empresa também focaliza suas estratégias na universidade corporativa, como forma de integrar as pessoas em diferentes setores. Reconhece que através da universidade corporativa aumenta a possibilidade de gerir melhor o conhecimento e a criar o seu próprio produto (software). A interação com universidades, reportando experiências de ensino, auxilia na concretização desta estraté gia. Neste contexto, a universidade corporativa disponibiliza plataforma de ensino à distância aos usuários, conforme ressalta o entrevistado:

“(...) a gente tem uma estrutura ai com a plataforma EAD muito bem estruturada, aonde todas as tecnologias e soluções e tudo que é de conhecimento novo, tanto na parte tecnológica e na parte de negócio, tanto para a parte, para a equipe técnica como para a equipe comercial, é gerenciada, é gerida pela universidade corporativa)". 
Para a empresa, a universidade corporativa constitui instância de disseminação do conhecimento por meio de treinamentos e de informação entre os colaboradores da empresa. Nesta perspectiva, concede acesso à informação aos colaboradores, na identificação de possibilidades tecnológicas e na criação de espaço de interação entre indivíduos de diferentes níveis de formação, como técnico s, graduados, mestres e doutores. Segundo o entrevistado, esta iniciativa cria um cenário integrador dentro da organização, uma vez que o acesso ao conhecimento é comum a todos, oferece mais recursos na criação de futuras soluções tecnológicas e no desenvolvimento de startups.

Para a empresa Delta, os elementos citados condizem com a formação de capacidades específicas aos membros organizacionais, que podem se tornar dinâmicas e gerarem novos produtos e serviços oferecidos. Contribui de forma decisiva para tal ocorrência, o fato da empresa estar inserida num sistema de inovação, em que além da interação com universidades dentre as quais a UFSC e UFCE, conta com outras instituições importantes de apoio, como as de financiamento do governo, em destaque o CNPq e o BNDES e regulamentação incentivadora, como a Lei do Bem.

Dentre as interações estabelecidas pela Ômega no âmbito de um sistema de inovação, a empresa dá preferência às relações que se firmam com o mercado, seguindo as orientações ao cliente, do que recorrer a outros participantes como universidades, institutos de pesquisa, governo e agentes de financiamento. $\mathrm{O}$ comportamento em atuar orientada ao cliente consiste na criação de segmentos específicos para atender nichos diferentes de empresas, como a área de manufatura, logística, jurídico, agroindustrial, entre outros. A empresa utiliza novidades do exterior e as adapta ao mercado brasileiro, em concordância com as necessidades dos usuários, que segundo o entrevistado, oferece serviço a um custo menor, tornando-se assim, um diferencial competitivo no mercado.

No Brasil, a empresa Ômega considera a universidade apenas como instância fornecedora de conhecimento para trabalhadores, e não como espaço que possibilita vínculos interativos voltados ao desenvolvimento da pesquisa. Nesta perspectiva, vale-se a empresa do conhecimento acadêmico e da experiência e de aprendizagem do trabalhador, para solucionar os problemas e criar produtos e serviços tecnológicos em interação com outras empresas ligadas ao setor de software. Segundo relato obtido na entrevista, este processo é constantemente realimentado pela frequência com que empresas privadas procuram a Ômega para fazer ajuste entre seus softwares e os softwares da empresa Ômega. Neste sentido, o entrevistado afirma:

\footnotetext{
"Eu vejo que a ÔMEGA não tem tanto histórico de fazer parcerias [...] com universidades. [...] tem muita gente que procura a ÔMEGA pra fazer parceria porque a gente tem muitos clientes [...], $e$ isso abre um leque muito grande pra essas empresas... tem várias empresas que buscam muito isso...então eu acho que é por isso que a ÔMEGA não procura tantas parcerias, [...]....as parcerias é que vêm até ela".
}

Tal posicionamento decorre de a empresa apresentar um volume significativo de clientes e valerse, para tanto, mais da relação produtor de software e usuário, do que interações com universidade e institutos de pesquisa, para desenvolvimento tecnológico de seus produtos e serviços. A trajetória de desenvolvimento percorrida contribui para este posicionamento, pois são décadas atuando no segmento de software. Construiu path dependence, que a permite utilizar dos benefícios que a interação com o usuário possibilita, através dos mecanismos de aprendizagem pelo uso.

Mas essa relação começa a mudar a partir 2010, quando a empresa estabelece parceria com duas universidades na Califórnia, EUA: A Universidade de Stanford e San Jose State (SJSU). A parceria com a Universidade de Stanford tem o objetivo de criar novos negócios no mercado americano, enquanto que o vínculo com a Universidade San Jose State possibilita realizar estudos a respeito do ambiente de desenvolvimento dos softwares, avaliando ferramentas e metodologias de criação de programas.

A empresa Lambda, já realizou no passado interação com as universidades. Naquela oportunidade, vínculos ocorreram para a realização de pesquisas de uso imediato. Uma vez atendida a demanda, encerrou-se o contrato firmado. A afirmação do entrevistado sinaliza neste sentido: "[...] a gente já teve algumas experiências, mas não temos nada efetivamente. [...]”. No momento, vínculos com o meio acadêmico através de projetos de pesquisa não ocorrem. 
Por sua vez, as relações estabelecidas pela empresa com a universidade estão vinculadas na área do ensino, por meio da frequência de seus trabalhadores nos cursos de graduação de uma universidade local. Assim como, por meio prioritário de contratação de pessoal vinculado a esta universidade. Uma particular característica desta interação é a disponibilidade dos produtos da empresa para processos de aprendizagem nos cursos de Administração da universidade local. Cita-se, como exemplo, o uso de software gerador de fluxo de caixa. O Entrevistado relata o seguinte:

\footnotetext{
“(...) a gente teve experiências no sentido de fornecer o nosso produto para que usassem no curso de administração; por exemplo, usando o produto da Lambda, isso a gente fez aqui na FURB, tínhamos até recentemente uma negociação acontecendo como Instituto Catarinense de Educação aqui em Blumenau, pra usar os produtos da Lambda como parte da aula, geralmente no curso de administração".
}

Por sua vez, uma das formas encontradas para buscar informações pela empresa ocorre por meio de consultorias. Estas são contratadas para obtenção de informações sobre as tendências do mercado que atendem às necessidades dos clientes. Assim como, realiza parcerias técnicas com empresas privadas, para complementar os produtos e/ou serviços oferecidos. Através destes mecanismos, as empresas trocam-se informações tecnológicas importantes visando alcançar objetivos comuns. Cita-se, como exemplo, uma situação em que a empresa parceira vende algumas ferramentas de apoio no produto e a Lambda atua na venda do software para controle.

\subsection{Análise Geral das Interações Estabelecidas e Capacidades de Absorção das Firmas Investigadas}

Como observam Zahra e George (2002), a empresa desenvolve a capacidade de absorção de conhecimento externo para orientar e completar o conhecimento interno no propósito de promover mudanças técnicas relevantes. Considerando este aporte, as empresas em estudo recorrem às universidades, para apoiarem o desenvolvimento de suas atividades. Um ponto comum em todas as empresas estudadas, refere-se à contratação de profissionais formados nas áreas de conhecimento da computação, engenharia, administração e sistema de informação pelas universidades, situadas, mas não só, no local e região em que se encontram.

As empresas - Alfa, Gama, Delta, Ômega e Lambda -, identificam e valorizam, de forma explícita, o conhecimento externo proveniente das universidades. Um dos procedimentos utilizados refere-se em criar condições para que seus trabalhadores obtenham titulação universitária. Mecanismos como, dispensa do trabalho nas horas do curso, pagamento de parte da mensalidade e sistema de promoção interna pós conclusão estimulam os trabalhadores à obtenção e atualização do conhecimento. Também, firmam-se convênios com universidades para seus trabalhadores realizarem cursos de pós-graduação, em área de interesse empresarial. Tais registros encontram-se em linha com os escritos de Cohen; Levinthal (1990 e 1994), expressos pelo reconhecimento empresarial do valor do conhecimento externo, obtidos pelos seus trabalhadores no propósito de aplica-lo em suas atividades.

Da mesma forma, as empresas - Alfa, Gama, Delta e Ômega - interagem com as universidades para desenvolvimento de pesquis a orientad as em solucionar problemas, aperfeiçoar sistemas, auxiliar na criação de novos produtos entre outros propósitos. Para além das capacidades de assimilação e absorção, conforme Zahra e George (2002), tais empresas demonstram as capacidades de transformação e exploração do conhecimento externo, recorrendo a parcerias com o meio acadêmico. Papel importante neste processo, são os vínculos formados pelas empresas com os laboratórios, departamentos e fundações da UFSC. Citam-se os arranjos efetuados pela empresa Gama com os laboratórios LISHA e LINSE e a fundação CERTI e os vínculos estabelecidos da empresa Alfa com o Instituto Stela, constituem referências.

Contribui de forma efetiva no proces so interativo das empresas com as universidades, o sistema de inovação nacional. Há registros do CNPq e da CAPES concederem recursos para pagamento de custos operacionais de pesquisa no propósito de incentivar os esforços inovativos. Assim como, apontamentos do BNDES e da Lei do Bem auxiliarem as empresas na montagem e modernização de laboratórios de P\&D. Tais ocorrências seguem as observações de Edquist (2001), que os integrantes de um sistema de inovação agem no propósito de criar condições para desenvolvimento e difusão do conhecimentos pró-inovação. Da 
mesma forma, tais ações institucionais estão em linha com os escritos de Van den Boschi; Volberda e Boer (1999), de que as empresas identificam, assimilam e exploram o conhecimento considerando as economias de custos e de escala que podem obter.

No desenvolvimento das interações das empresas de software e as universidades deve-se considerar, conforme Zahra; George (2002) e Xia; Riper (2008), os resultados alcançados. As empresas consideram no âmago dos processos de assimilação, absorção, transformação e exploração do conhecimento, a taxa e a efetividade dos processos resultantes. Os resultados expressam linhas do progres so técnico obtidas a partir de esforços mútuos realizados. Constroem-se path-dependencies relacional e linhas de processos de aprendizagem. Prova disto é a temporalidade das interações. As empresas Alfa e a Gama realizam parcerias com universidades, desde a década de 1990. A Delta mantém parcerias a partir da década de 2000. E, mais recente, a Lambda sustenta a interação desde 2009 e a Ômega realiza vínculos pós o ano 2010.

Quadro 2: Principais resultados da interação das empresas do setor de software selecionadas de Santa Catarina com Universidades, 2016.

\begin{tabular}{|c|c|c|c|c|}
\hline Empresas & Universidades & $\begin{array}{c}\text { Formação de } \\
\text { Mão-de-obra }\end{array}$ & $\begin{array}{c}\text { Realização } \\
\text { de Pesquisa }\end{array}$ & $\begin{array}{c}\text { Resultados Relevantes da Interação Universidade- } \\
\text { Empresa }\end{array}$ \\
\hline Alfa & UFSC & $\mathrm{X}$ & $\mathrm{X}$ & $\begin{array}{c}\text { Desenvolvimento de tecnologia para segurança de } \\
\text { dados em nuvem }\end{array}$ \\
\hline Gama & UFSC, UFCE e SENAI & $\mathrm{X}$ & $\mathrm{X}$ & $\begin{array}{c}\text { Criação do taquígrafo que transcreve } \\
\text { automaticamente o áudio }\end{array}$ \\
\hline Ômega & $\begin{array}{c}\text { Stanford e San Jose } \\
\text { State }\end{array}$ & $\mathrm{X}$ & $\mathrm{X}$ & $\begin{array}{c}\text { Desenvolvimento de ferramentas e metodologias em } \\
\text { novos softwares }\end{array}$ \\
\hline Lambda & FURB e IFSC & $\mathrm{X}$ & - & $\begin{array}{c}\text { Uso de software em processos de aprendizagem dos } \\
\text { cursos de graduação }\end{array}$ \\
\hline
\end{tabular}

Fonte: Pesquis a de Campo.

Os produtos inovadores gerados a partir das interações dos meios empresarial e o acadêmico, comprovam a virtuosidade das relações firmadas, conforme Quadro 2. Na empresa Alfa, a parceria com a UFSC resulta no desenvolvimento de tecnologia para segurança de dados em nuvem. Na empresa Gama, a interação possibilita a criação do taquígrafo que transcreve automaticamente o áudio. Na empresa Delta, vínculos firmados possibilitam seus pesquisadores obterem os títulos de mestre e doutor, bem como, resultam em auxílio no desenvolvimento do projeto próprio de universidade corporativa. Na empresa Ômega, as parcerias firmadas com as Universidades de Stanford e de San Jose State, Califórnia, EUA, possibilitam o desenvolvimento de ferramentas e metodologias para criação de novos softwares. E, na empresa Lambda, a interação com a FURB permitem o uso de seus produtos nos processos de aprendizagem dos estudantes universitários.

Os resultados inovativos apresentados devem ser considerados à luz dos procedimentos adotados pelas empresas. Em linha com os escritos de: Todorova; Durisin (2007), as empresas em estudo, reconhecem e dão importância ao conhecimento externo, realizando vínculos interativos com suas fontes geradoras. Bem como, criam condições do mésticas para assimilar o conhecimento externo e inter-relacionálo com o interno, em processos de mudança das estruturas cognitivas existentes. Na mesma perspectiva, a quase totalidade das empresas pesquisadas empresas posiciona favoravelmente aos escritos de Liao, $\mathrm{Wu}$, Hu e Tsuei (2009): primeiro, por eleger o processo inovativo como caminho para obtenção de vantagem competitiva (com exceção de apenas uma que privilegia a concorrência via preço e valoriza relativamente menos as interações com universidades do que as demais); e, segundo por contarem com condições infraestruturais internas, dentre as quais laboratórios, equipamentos, recursos financeiros e pessoal qualificado para absorver o conhecimento externo.

Portanto, observou-se nas interações identificadas que a Universidade exerce tanto sua função tradicional de ensino, quanto suas funções de pesquisa e extensão (no papel de ensino corporativo, por exemplo). Destaca-se também que as empresas investigadas são de grande porte, algo que resulta em viés nos achados da pesquisa, no sentido de valorizarem e usarem fontes externas de conhecimento, algo pouco observado na Pesquisa de Inovação (PINTEC) - do Instituto Brasileiro de Geografia e Estatística (IBGE) - 
que informa o padrão de inovação das firmas brasileiras. Há certo consenso na literatura que empresas de grande porte, na média, possuem melhores condições para absorver conhecimentos, interagir com outros agentes do sistema de inovação e inovar. Por fim, vale ressaltar o uso complementar de fontes de apoio à inovação, como, por exemplo, o instrumento legal da Lei do Bem, ao mecanismo de interação com universidades. Isso parece informar que a capacidade de absorção de conhecimento externo das firmas também as permite usufruírem de diferentes incentivos à atividade inovativa.

\section{Considerações Finais}

O presente estudo demonstra que as empresas de grande porte do setor de software selecionadas de Santa Catarina - fazem uso da capacidade de absorção de conhecimento externo, para promoverem melhores condições internas de desenvolvimento de suas atividades. Cientes que se encontram em um setor baseado em ciência, elegem ações - estratégias - voltadas à busca deste tipo de conhecimento no ambiente institucional que as cercam. A presença de integrantes do sistema de inovações - universidades, governo, instituições de fomento e sistema financeiro - auxilia na concretização deste propósito.

As empresas - Alfa, Beta, Gama, Ômega e Lambda - por elegerem o conhecimento como um dos principais ativos, recorrem às universidades, em grande monta localizadas em Santa Catarina - UFSC, FURB, UDESC, UNISUL, IFSC entre outras -, para desenvolvimento de interações. Algumas complementam suas interações com o meio acadêmico, além do espaço estadual. No geral, reconhecem a universidades, como espaços de repositórios de conhecimentos científico e tecnológico, cujos serviços prestados nas áreas de ensino, pesquisa e extensão são considerados relevantes.

Neste sentido, sintetizam-se os principais resultados característicos das interações universidadeempresa realizadas no segmento de software estadual:

a) Vínculos interativos de longo prazo, com registro de contratos com algumas empresas há mais de 2 décadas;

b) Contribuição virtuosa das universidades e seus laboratórios no desenvolvimento técnico e inovativo de produtos e serviços das empresas;

c) Formação de mão-de-obra qualificada pelas universidades para atuação nas áreas da tecnologia de informação e de administração das empresas;

d) Prestação de serviços pelas empresas nos laboratórios e estruturas administrativas das universidades para aperfeiçoamento de suas atividades;

e) Desenvolvimento de mentalidade de esforços cooperativos para busca inovativa pelos meios empresariais e universitários;

f) Esforços das empresas na criação de infraestrutura tecnológica - laboratório, pessoal e recursos - para melhor desenvolver a capacidade de absorção;

g) Incorporação de novas procedimentos de busca e de rotina em operações voltadas ao desenvolvimento inovativo pelas empresas; e,

h) Reconhecimento da contribuição da universidade na obtenção de registros de patentes de produtos e serviços inovadores pelas empresas.

Demonstra-se, enfim, que o processo de construção do conhecimento de uma empresa deve levar em consideração não somente a base interna, mas as fontes externas. Este condicionante soma-se à consideração que o processo inovativo é sistêmico, envolvendo vários atores. Logo, não é sem razão que os esforços inovativos são cada vez mais institucionalizados, considerando os recursos da própria empresa, ativos tangíveis e intangíveis destinados a sua viabilização, mas também a existência de um sistema de inovação, cujas instituições, com funções distintas, contribuem de forma ativa para o desenvolvimento do sistema econômico de um país.

\section{Referências}

ALVIM, P. C.; Cooperação universidade- empresa: da interação a realidade. Interação Universidade Empresa. Brasilia: IBICT, 1998. 
ARZA, V. Channels, benefits and risks of public-private interactions for knowledge transfer: conceptual framework inspired by Latin America. Science and Public Policy, v.37, n.7, p.473-484, aug.2010.

BETTS, S. C.; SANTORO, M. D. Somewhere between markets and hierarchies: controlling industry university relationships for success. Academy of Strategic Management Journal, v.10, n.1, 2011.

BISHOP, K.; D'ESTE, P.; NEELY, A. Gaining from interactions with universities: multiple methods for nurturing absorptive capacity. Research Policy. Volume 40, Issue 1, February 2011, Pages 30-40. https://doi.org/10.1016/j.respol.2010.09.009

BRUNEEL, J.; D'ESTE, P.; SALTER, A. Investigating the factors that diminish the barriers to universityindustry collaboration. Research Policy, v.39, p.858-868, 2010.

CASSIOLATO, J. E.; GADELHA, C. G.; ALBUQUERQUE, E.; BRITTO, J. A relação universidade e instituições de pesquisa com o setor industrial: uma análise de seus condicionantes. IE/UFRJ, 1996. Mimeografado.

CHANG, H.; HOU, J. J.; LIN, S. A multi-cases comparative approach on forming Elements of dynamic capability. The International Journal of Organizational Innovation, v. 5, n. 4, p. 52-64, 2013.

CHIVA, R.; ALEGRE, J. Organizational learning and organizational knowledge towards the integration of two approaches. Management Learning, v. 36, n. 1, p. 49-68, 2005.

COHEN, W. M.; LEVINTHAL, D. Empirical studies of innovation and market structure. Handbook of Industrial Organization, v. 2, p. 1059-1107, 1989.

COHEN, W.; LEVINTHAL, D. Absorptive capacity: a new perspective on learning and innovation. Administrative science quarterly, v. 35, n. 1, p. 128-152, 1990.

COHEN, W. M.; LEVINTHAL, D. A. Fortune favors the prepared firm. Management Science, v. 40, n. 2, p. 227-251, 1994.

COHEN, W. M.; NELSON, R. R.; WALSH, J. P. Links and impacts: the influence of public research on industrial R\&D. Management Science, v.48, n.1, p.1-23, jan.2002.

DUTRÉNIT, G.; ARZA, V.; Channels and benefits of interactions between public research organisations and industry: comparing four Latin American countries, Science and Public Policy, Volume 37, Issue 7, 1 August 2010, Pages 541-553, https://doi.org/10.3152/030234210X512043

EDQUIST, C. Systems of innovation: technologies, institutions and organizations. London: Pinter, 1997.

EDQUIST, C. Systems of innovation for development (SID), Background Paper for Chapter I: "Competitiveness, innovation and learning: analytical framework" for the UNIDO World Industrial Development Report (WIDR), 2001. Innovation, p. 1-42, 2001.

EISENHARDT, K. M.; MARTIN, A. J. Dynamic capabilities: what are they? Strategic Manage ment Journal, v. 21, p. 1105-1121, 2000.

FEDERAÇÃO DAS INDÚSTRIAS DO ESTADO DE SANTA CATARINA. FIESC: relatório anual 2014 / Federação das Indústrias do Estado de Santa Catarina. Florianópolis, 2015. 44 p.: il. color.

FLEURY, A. L. Alinhando objetivos estratégicos e processo de desenvolvimento em empresas de software. 201p. Tese (Doutorado em Engenharia). Escola Politécnica, Universidade de São Paulo USP. São Paulo. 2007.

HUBER, G. P. Organizational learning: the contributing processes and the literatures. Organization Science, v. 2, n. 1, p. 88-115, 1991.

JANSEN, J. J. P.; VAN DEN BOSCH, F. A. J.; VOLBERDA, H. W. Managing potential and realized absorptive capacity: how do organizational antecedents matter? Academy of Manage ment Journal, v. 48, n. 6, p. 999-1015, 2005. 
KIM, D. H. The link between individual and organizational learning. The strategic management of intellectual capital, p. 41-62, 1998.

LANE, P. J.; KOKA, B. R.; PATHAK, S. The reification of absorptive capacity: a critical review and rejuvenation of the construct. Academy of Management Review, v. 31, n. 4, p. 833-863, 2006.

LEE, Y. S. The sustainability of university-industry research collaboration: an empirical assessment.

Journal of Technology Transfer, v.25, p.111-133, 2000.

LEMOS, D. C. A interação universidade-empresa para o desenvolvimento inovativo sob a perspectiva institucionalista-evolucionária: uma análise a partir do sistema de ensino superior em Santa Catarina. Tese (Doutorado em Administração) - Programa de Pós-Graduação em Administração, Centro Sócio Econômico, Universidade Federal de Santa Catarina (UFSC), Florianópolis, 2013.

LIAO, S. H. et al. Knowledge acquisition, absorptive capacity and innovation capability: an empirical study of Taiwan's knowledge-intensive industries. World Academy of Science, Engineering and Technology, v. 53, p. 160-167, 2009.

MALERBA, F.; L. ORSENIGO, L. L'accumulazione delle capacita tecnologiche nell industria italiana (1969-1984), In: Filippini, L. (ed.), Innovazione tecnologica e servizi alle imprese, Milán, Italia, Franco Angeli. 1993.

MEYER-KRAMER, F.; SCHMOCH, U. Science-based technologies: university-ind ustry interactions in four fields. Research Policy, v.27, n.8, p.835-851, 1998. https//doi.org/10.1016/S0048-7333(98)00094-8

MUELLER, S. P. M. A comunicação científica e o movimento de acesso livre ao conhecimento. Ciência da Informação., Brasilia, v. 35, n. 2, p. 27-38, maio/ago. 2006

MOWERY, D. C. Economic theory and government technology policy. Policy Sciences, v. 16, n. 1, p. $27-$ 43, 1983.

MOWERY, D. C.; OXLEY, J. E. Inward technology transfer and competitiveness: the role of national innovation systems. Cambridge Journal of Economics, 19, 67-93. 1995.

MOWERY, D. C.; OXLEY, J. E.; SILVERMAN, B. . Strategic alliances and interfirm knowledge transfer. Strategic Management Journal, v. 17, n. Winter Special Issue, p. 77-91, 1996.

NELSON, R. R.; WINTER, S. G. The schumpeterian trade off revisited. The American Economic Review, v. 72, n. 1, p. 114-132, 1982.

NELSON, R; WINTER, S. Uma teoria evolucionaria da mudança econômica. Unicamp, 2006.

OSHRI, I.; PAN, S. L.; NEWELL, S. Managing trade-offs and tensions between knowledge management initiatives and expertise development practices. Management Learning, v. 37, n. 1, p. 63-82, 2006.

PAVITT, K. What we know about the strategic management of technology. California Management Review. University of California Press. 1990

PERKMANN, M.; WALSH, K. University-ind ustry relationships and open innovation: towards a research agenda. International Journal of Management Reviews, v.9, p.259-280, 2007.

PISANO, G. P.; TEECE, D. J. How to capture value from innovation: Shaping intellectual property and industry architecture. California Management Review, v. 50, n. 1, p. 278-296, 2007.

SEGATTO-mendes, A. P., SBRAGIA, R. O processo de cooperação universidade-empresa em universidades brasileiras. Revista de Administração, 37(4), 58-71. 2002.

$<$ http $/ /$ www.spell.org.br/documento s/ver/16709/o-processo-de-cooperacao-universidade-empresa-emuniversidades-brasileiras/i/pt-br>.

TODOROVA, G.; DURISIN, B. Absorptive capacity: valuing a reconceptualization. Academy of Management Review, v. 32, n. 3, p. 774-786, 2007. 
VAN DEN BOSCH, F. A. J.; VOLBERDA, H. W.; BOER, M. Coevolution of firm absorptive capacity and knowledge environment: organizational forms and combinative capabilities. Organization Science. 10:5, 551-568. 1999.

WINTER, S. G. The satisficing principle in capability learning. Strategic Management Journal, v. 21, p. 981-996, 2000.

XIA, T.; ROPER, S. From capability to connectivity-absorptive capacity and exploratory alliances in biopharmaceutical firms: A US-Europe comparison. Technovation, v. 28, n. 11, p. 776-785, 2008.

ZAHRA, S. A.; GEORGE, G. Ab sorptive capacity: a review, reconceptualization, and extension. Academy of Management Review, v. 27, n. 2, p. 185-2003, 2002. 